THE ASTROPHYSICAL JOURNAL, 523:838-848, 1999 October 1

(C) 1999. The American Astronomical Society. All rights reserved. Printed in U.S.A

\title{
MAGNETIC RECONNECTION SOLUTIONS IN THE PRESENCE OF MULTIPLE NULLS
}

\author{
I. J. D. Craig, R. B. Fabling, J. Heerikhuisen, and P. G. Watson \\ Department of Mathematics, University of Waikato, Private Bag 3105, Hamilton, New Zealand \\ Received 1998 September 14; accepted 1999 May 11
}

\begin{abstract}
It is known that exact analytic solutions can be constructed for incompressible magnetic reconnection in three space dimensions. In the case of an isolated $X$-point null, there are two types of reconnection solutions, namely, "spine" and "fan" models, which depend on the form of the $X$-point disturbance. However, such models cannot describe multiple null "separator" reconnection, for which there is independent observational evidence. Here we show that the spine formalism naturally extends to the case of multiple null fields. Solutions showing the characteristics of fan, spine, and separator are described, and a discussion is given of their energy dissipation properties. We demonstrate a family of multiple null, fast reconnection solutions and point out that the classical Sweet-Parker dissipation rate is the slowest that can be achieved with the present models.
\end{abstract}

Subject headings: MHD - Sun: magnetic fields

\section{INTRODUCTION}

Although magnetic reconnection is recognized as a key mechanism in the evolution of highly conducting plasmas, there are many aspects of reconnection theory that remain poorly developed. A persistent puzzle is accounting for the rapid magnetic collapse of the solar flare (e.g., Parker 1979). Early flare studies were restricted mainly to steady-state magnetic merging in plasmas containing an isolated neutral point (Forbes \& Priest 1987). This work highlighted the central role played by magnetic separatrices - lines and surfaces formed by field lines threading the null. Separatrices form sites of strong current accumulation and enhanced ohmic dissipation in the plasma. More recent work involving multiple nulls (e.g., Lau \& Finn 1990; Priest \& Titov 1996) also suggests that the form of the reconnection may be determined by the details of the separatrix topology. Apart from theory, solar observations from Yohkoh (Demoulin et al. 1996) provide independent evidence of multiple null "separator reconnection."

It is now known that exact analytic solutions for incompressible magnetic reconnection can be constructed in two and three space dimensions (Craig \& Henton 1995; Craig \& Fabling 1996; Watson \& Craig 1997a; Watson \& Craig 1997b). These solutions possess many of the characteristics required by a "fast" reconnection mechanism. In particular, they predict a collapse of the magnetic field to smaller and smaller length scales as the plasma resistivity is reduced - a collapse that remains uncompromised by the addition of time-dependent and viscous effects (Craig \& Watson 1998). At present such models are restricted to isolated $X$-point nulls for which the separatrix structure is particularly simple. In this paper we show that the formalism employed in developing single null models extends naturally to describe certain classes of multiple null solutions

Consider, for a moment, the separatrix structure of a single $X$-point null in three dimensions, as illustrated in Figure 1. There is an isolated "fan" plane, which is threaded by a single field line called the "spine" curve (in the nomenclature of Priest \& Titov 1996). The null is said to be positive (negative) if the spine defines a field line which exits (enters) the null (compare Greene 1988 and Lau \& Finn 1990). Evidently, the form of the reconnection depends upon how the separatrices are distorted by magnetic disturbances. "Spine current reconnection," involving quasicylindrical currents aligned to the spine, is driven by distortions of the fan (Craig \& Fabling 1996). By contrast, "fan current reconnection" involves displacements of the spine and leads to a narrow current sheet overlying the fan surface.

In the case of multiple nulls, the separatrix structure is far more complicated. An example involving a central null surrounded by three satellite nulls is shown in Figure 2. Apart from multiple spine curves, individual fan planes can intersect to form "separator" lines (see Fig. 2a). Kinematic considerations suggest that reconnection can be associated with the formation of current ribbons on separators. Additional support is provided by X-ray observations, which show that bright points may be produced by high-current separatrix layers (Demoulin et al. 1996). However, unlike spine and fan models for an isolated null, there is no exact description of separator reconnection (see Longcope \& Cowley 1996; Longcope 1998).

The aim of this paper is to develop reconnection solutions for magnetic fields comprising multiple nulls. One motivation - aside from theoretical curiosity - is to provide a detailed description of separator reconnection. In contrast to previous kinematic studies of multiple null reconnection (e.g., Lau \& Finn 1990; Priest \& Titov 1996), our aim is to provide a fully self-consistent treatment of the induction and momentum equations. There is also the possibility that multiple null solutions (e.g., Parnell 1996; Priest 1997) might allow more favorable energy release scalings than single null models. In fact, Watson \& Craig (1997b) have already shown that multiple nulls can allow different scalings for the energy dissipation rate.

In $\S 2$ we present the spine and fan formulation for constructing exact reconnection solutions (Craig \& Fabling 1996). We point out that, although multiple null points cannot be accommodated within the fan current formulation, the spine formulation is considerably more flexible. We go on in $\S 3$ to discuss the general properties of multiplenull solutions and explore a particular example - the model of Figure 2-involving a ring of nulls surrounding a central neutral point. In $\S 4$ we show that the multiple null formalism is capable of representing, quantitatively, all the basic 


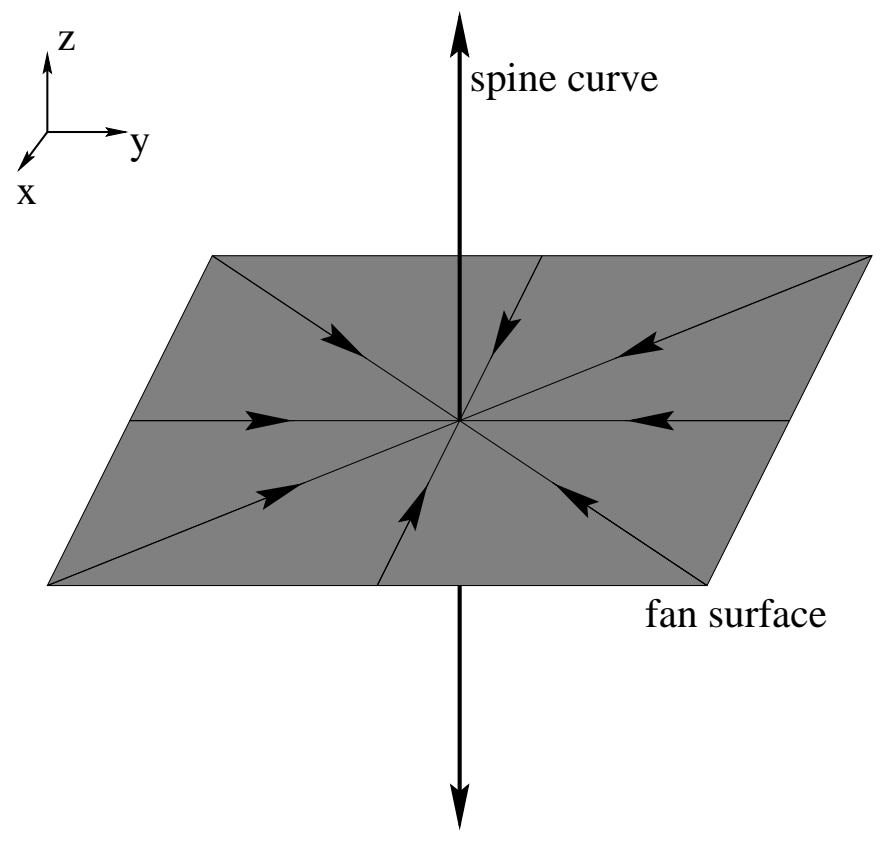

FIG. 1.- Schematic diagram of the separatrix structure of a single positive null showing both the spine curve and the fan plane.
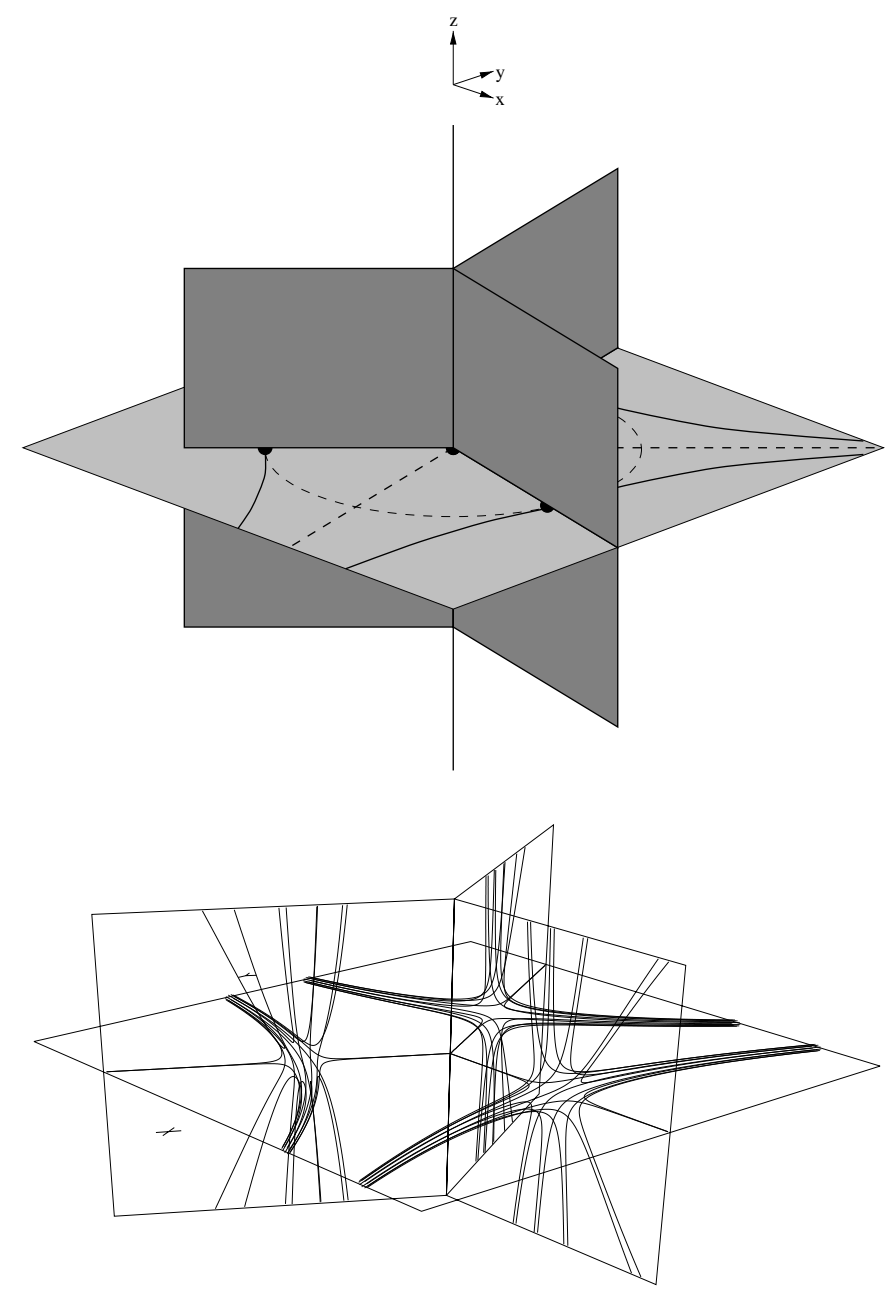

FIG. 2.-Top: Separatrix structure of a background potential field with cylindrical symmetry for $m=3$. Bottom: actual field line structure for the satellite nulls. forms of reconnection discussed in the literature. Our conclusions are summarized in $\S 5$.

\section{FORMULATION}

\subsection{MHD Equations}

We assume that the plasma is governed by the steadystate momentum and induction equations for an incompressible, resistive plasma. This system is conveniently written in the nondimensional form

$$
\begin{aligned}
& \nabla \times(\omega \times v)=\nabla \times(J \times B), \\
& (v \cdot \nabla) B-(B \cdot \nabla) v=\eta \nabla^{2} B,
\end{aligned}
$$

where the magnetic and velocity fields satisfy $\nabla \cdot \boldsymbol{B}=$ $\nabla \cdot v=0$, and the current density and fluid vorticity are given by

$$
J=\nabla \times B, \quad \omega=\nabla \times v .
$$

We seek solutions for the $\boldsymbol{B}$ and $\boldsymbol{v}$ fields inside the unit cube $-1 \leq x, y, z \leq 1$.

The resistive losses of the plasma provide the only source of dissipation. In the present formulation the resistivity $\eta$ is very small, on the order of $10^{-12}$ for the solar corona, and so the ohmic dissipation rate

$$
W_{\eta}=\eta \int J^{2} d V
$$

can be significant only in the presence of steep gradients in the magnetic field. A central aim of reconnection theory is to derive "fast" solutions, for which $W_{\eta}$ scales independently of any positive power of the plasma resistivity. It follows that we are interested only in those solutions that exhibit increasingly small length scales with reductions in the resistivity.

\subsection{Form of the Field and Flow}

To determine analytic solutions we exploit the symmetry in the magnetic and velocity fields. We consider the superposition

$$
\boldsymbol{v}(\boldsymbol{x})=\boldsymbol{P}+\lambda \boldsymbol{Q}, \quad \boldsymbol{B}(\boldsymbol{x})=\lambda \boldsymbol{P}+\boldsymbol{Q},
$$

where $\lambda$ is a constant and $\boldsymbol{P}$ is some suitably chosen global field. The "background" field $\boldsymbol{P}$ contains no small length scales. For a given $\boldsymbol{P}$ the disturbance field $\boldsymbol{Q}$ is determined by solution of the resistive MHD equations (1)-(2). We expect the $Q$-field to contain small length scales determined by the level of the plasma resistivity.

In this paper we require that $\boldsymbol{P}$ defines a current-free equilibrium:

$$
\boldsymbol{\nabla} \times \boldsymbol{P}=0, \quad \boldsymbol{\nabla} \cdot \boldsymbol{P}=0 .
$$

More general "splittings" of the $\boldsymbol{B}$ and $\boldsymbol{v}$ fields are possible, which allow time-dependent viscous solutions to be developed (Craig \& Watson 1998). However, forms (5) and (6) are sufficient for the present purposes. In particular, equation (6) admits the prototype isolated $X$-point solution

$$
\boldsymbol{P}_{X}=\alpha[\kappa x \hat{\boldsymbol{x}}+(1-\kappa) y \hat{\boldsymbol{y}}-z \hat{\boldsymbol{z}}] .
$$

The parameter $\alpha$ governs the strength of the field, and $\kappa$ governs the degree of anisotropy. Note that for $0 \leq \kappa \leq 1$ the null is oriented so that the spine lies along the $z$-axis, in contrast to Craig \& Fabling 1996, who align the spine with 
the $x$-axis. For $\alpha>0$ the null is negative since the spine field line, aligned to the $z$-axis, enters the null.

\subsection{Spine and Fan Disturbance Fields}

The form of the disturbance field $\boldsymbol{Q}$ is limited mainly by the momentum equation. We find that $\boldsymbol{Q}$ must satisfy

$$
\boldsymbol{\nabla} \cdot \boldsymbol{Q}=0, \quad \boldsymbol{\nabla} \times[(\boldsymbol{\nabla} \times \boldsymbol{Q}) \times \boldsymbol{Q}]=0 .
$$

There are two distinct forms for $\boldsymbol{Q}$, both involving straight field lines, which allow analytic solutions to be developed. Either $\boldsymbol{Q}$ is dependent only on (the spine axis) $z$ and

$$
\boldsymbol{Q}_{F}(z)=X(z) \hat{\boldsymbol{x}}+Y(z) \hat{\boldsymbol{y}},
$$

or there is the single component form

$$
\boldsymbol{Q}_{S}(x, y)=Z(x, y) \hat{z},
$$

in which the field amplitude $Z(x, y)$ varies over the fan surface. These are the fan and spine forms for the disturbance field $Q$ discussed by Craig \& Fabling (1996).

In the following sections we shall consider only the spine formulation in equation (10). For an isolated null, as given in equation (7), current accumulation about the neutral point requires outflow along the spine axis, that is, $\alpha<0$, corresponding to a positive null. As far as multiple null solutions are concerned, it is easy to verify, following Fabling (1997), that the fan formulation allows only trivial variants of the isolated $X$-point null in equation (7). The spine models incorporate not only fully two-dimensional disturbance fields but also, in a certain sense, the fan solutions (see $\S 4.3$ ). This follows by noting that a single fan component $\boldsymbol{Q}_{F}=X(z) \hat{\boldsymbol{x}}$ can be modeled by taking the restricted spine form $Q_{S}=Z(x) \hat{z}$ and interchanging the $x$ and $z$ coordinates. Thus general fan models can always be constructed by the superposition of two one-variable spine solutions.

\subsection{Cartesian Spine Equations}

To confirm that the spine formulation admits multiple null solutions we substitute the form in equation (5) into the induction equation assuming the spine form in equation (10). The Cartesian components of $\boldsymbol{P}(x)=\alpha\left(P_{1}, P_{2}, P_{3}\right)$ must satisfy

$$
\begin{gathered}
-P_{3, z} Z+P_{1} Z_{x}+P_{2} Z_{y}=\bar{\eta}\left(Z_{x x}+Z_{y y}\right), \\
P_{1, z} Z=0, \quad P_{2, z} Z=0,
\end{gathered}
$$

where

$$
\bar{\eta}=\frac{\eta}{\alpha\left(1-\lambda^{2}\right)},
$$

and subscripted letters indicate partial differentiation. The $y$ - and $z$-components of the induction equation imply $P_{1}=$ $P_{1}(x, y), \quad P_{2}=P_{2}(x, y)$, which together with the $x$ component of the induction equation and the chosen disturbance form $Z(x, y)$ imply that $P_{3}$ must be a linear function of $z$. These conditions along with the constraint equations (6) yield

$$
P_{3}=-\sigma z, P_{1, x}+P_{2, y}=\sigma, \quad P_{1, y}=P_{2, x},
$$

where $\sigma$ is a global constant.

It is easy to see that the $x$ - and $y$-components of $\boldsymbol{P}$ are not restricted to linear functions. By taking

$$
P_{1}=\gamma \sigma x+H_{1}(x, y), \quad P_{2}=(1-\gamma) \sigma y+H_{2}(x, y),
$$

with $\gamma$ constant, we note that equation (13) reduces to Cauchy-Riemann equations for the conjugate harmonic pair $H_{1}$ and $H_{2}$. This result is exploited in $\S 3.2$, when we consider the cylindrical coordinate representation of the spine equation (see eq. [14]).

Finally we mention that the limit $\sigma=0$ has little physical interest. In this case the disturbance field $Z$ is advected only by transverse components of the flow. Since flow components normal to the field cannot stretch the field lines and magnify the field, there is no possibility of fast resistive dissipation for $\sigma=0$ (Fabling \& Craig 1996). In fact, for an isolated null located at the origin (given by eq. [7], say), the physically interesting case is $\sigma>\kappa>0$ and $\alpha$ negative. The $z$-axis then forms a spine curve aligned to the exhaust axis of the fluid. The disturbance field is independent of distance along the spine, and the current density,

$$
\boldsymbol{J}(x, y)=\nabla \times \boldsymbol{Q}=Z_{y} \hat{\boldsymbol{x}}-Z_{x} \hat{\boldsymbol{y}},
$$

tends to localize in tubular structures around the spine. For the multiple null solutions dealt with in the analysis to follow, we can relax these assumptions somewhat and take $\sigma>0, \alpha<0$.

\subsection{Spine and Fan Energy-Release Models}

It is important to stress that, in the case of an isolated $X$-point null (eq. [7]), the energy-release properties of the spine and fan solutions can be quite different. Energetically the fan solutions are considerably more favorable for fast flarelike energy release than spine models based on axisymmetric background fields (as detailed by Craig, Fabling, \& Watson 1997). This is a consequence of the geometry of the localized current structures that develop on the outflow separatrices of the fluid. In particular, the slab geometry of current sheets localized to the fan plane $z=0$ can accumulate appreciably more magnetic energy than tubular current structures aligned to the spine axis (Craig et al. 1997).

In the present formulation only spinelike disturbances can be used to generate multiple null solutions. Does this mean that multiple null models constructed in this way are necessarily unfavorable as energy-release models? This question is answered in $\S 4$, where we show that multiple null solutions obtained via the spine formulation can manifest aspects of fan, spine, and separator reconnection.

\section{MULTIPLE NULL SOLUTIONS}

\subsection{Introduction}

To make further progress it is convenient to assume that the background field $\boldsymbol{P}$ comprises a central null at the origin surrounded by satellite nulls in the plane $z=0$. In this way we can exploit the rotational symmetry of the problem about the spine axis.

We recall from $\S 2.4$ that departures from linearity in $\boldsymbol{P}$ involve the superposition of conjugate harmonic functions to the Cartesian components $P_{1}$ and $P_{2}$. In $\S 3.2$ we formulate the general problem but concentrate, for simplicity, on adding a single nonlinear component to $\boldsymbol{P}$ in $\S 3.3$. An analytic argument is then used in $\S 3.4$ to show that large currents are expected to localize on the fan planes of the satellite nulls. However, detailed resistive simulations suggest that the energy dissipation scalings do not depart significantly from the single null spine model. Solutions 
involving more general nonlinear contributions will be introduced in $\S 4$.

\subsection{Cylindrical Formulation of Multinull Solutions}

We replace $(x, y)$ by $(r, \theta)$ coordinates,

$$
r=\sqrt{x^{2}+y^{2}}, \quad \tan (\theta)=\frac{y}{x},
$$

and set

$$
\boldsymbol{P}=\alpha\left[P_{1}(r, \theta) \hat{\boldsymbol{r}}+P_{2}(r, \theta) \hat{\boldsymbol{\theta}}-\sigma z \hat{\boldsymbol{z}}\right], \quad \boldsymbol{Q}=Z(r, \theta) \hat{\boldsymbol{z}} .
$$

The divergence and curl conditions (eq. [6]) on the background field now imply

$$
\begin{aligned}
\frac{1}{r}\left(r P_{1}\right)_{r}+\frac{1}{r} P_{2, \theta}-\sigma & =0, \\
\frac{1}{r}\left(r P_{2}\right)_{r}-\frac{1}{r} P_{1, \theta} & =0 .
\end{aligned}
$$

These equations have solutions of the form

$$
\begin{gathered}
P_{1}=\frac{\sigma}{2} r+\sum_{k=2}^{\infty} \mu_{k} r^{k-1} \cos (k \theta), \\
P_{2}=-\sum_{k=2}^{\infty} \mu_{k} r^{k-1} \sin (k \theta) .
\end{gathered}
$$

The induction equation reduces to

$$
\sigma Z+P_{1} Z_{r}+\frac{P_{2}}{r} Z_{\theta}=\bar{\eta}\left[\frac{1}{r}\left(r Z_{r}\right)_{r}+\frac{1}{r^{2}} Z_{\theta \theta}\right],
$$

where $\bar{\eta}$ is given by equation (12). In the case where $P_{1}$ and $P_{2}$ are linear in $r$, we can remove the explicit dependence on $\bar{\eta}$ from the induction equation by letting $r \rightarrow r /|\bar{\eta}|^{1 / 2}$. It follows that there exists a universal small length scale $r \sim$ $|\bar{\eta}|^{1 / 2}$ in the disturbance field $Z$. We conclude that the presence of multiple small scales in $Z$ requires the admission of nonlinear components in the background field (see Watson \& Craig 1997b).

\subsection{Simple Nonlinear Nulls}

Let us consider the contribution of a single nonlinear component in the summation in equation (17). Taking $\sigma=1$ the explicit form of $\boldsymbol{P}$ is

$$
\boldsymbol{P}=\alpha\left\{\left[\frac{r}{2}+\mu r^{m-1} \cos (m \theta)\right] \hat{r}-\mu r^{m-1} \sin (m \theta) \hat{\boldsymbol{\theta}}-z \hat{\boldsymbol{z}}\right\} .
$$

In Cartesian coordinates we have that

$$
\begin{aligned}
& \boldsymbol{P}=\alpha\left\{\left[\frac{x}{2}+\mu \operatorname{Re}\left(w^{m-1}\right)\right] \hat{\boldsymbol{x}}+\left(\frac{y}{2}-\mu \operatorname{Im}\left(w^{m-1}\right)\right) \hat{\boldsymbol{y}}-z \hat{\boldsymbol{z}}\right\}, \\
& w=x+i y,
\end{aligned}
$$

and so, for $m=2$, the background field reduces to $\boldsymbol{P}=$ $\alpha\left[\left(\frac{1}{2}+\mu\right) x,\left(\frac{1}{2}-\mu\right) y,-z\right]$. This is just the $X$-point field (eq. [7]) with $\kappa=\frac{1}{2}+\mu, \sigma=1$. Obviously we must take $m \geq 3$ to achieve nonlinear solutions.

As already mentioned, the separatrix structure of the background field $\boldsymbol{P}$ provides an indication of the nature of the reconnection. For nonlinear nulls we can linearize the field in the vicinity of each null and determine the resultant eigenstructure (see the Appendix). In the present case there is a special null at the origin whose spine extends along the $z$-axis and whose fan lies in the plane $z=0$. This central null has satellite nulls that lie in a ring determined by the equations

$$
r^{m-2}=\frac{1}{2|\mu|}, \quad \theta=\left\{\begin{array}{ll}
2 k \pi / m, & \mu<0, \\
(2 k+1) \pi / m, & \mu>0,
\end{array} z=0,\right.
$$

as shown in Figure 2. The satellite spines lie in the fan of the central null; their fans extend out of the $z=0$ plane and intersect along the central spine axis $x=y=0$. Adjacent nulls on the circle are separated by a radial field line (Fig. $2 a$, dashed line) that pierces the central null. Note the absence of mutual null-null lines for the satellites. A detailed field line plot is shown in Figure $2 b$.

\subsection{Singular Ideal Solutions}

In the absence of resistivity we expect the induction equation (18) to contain singularities corresponding to infinite current densities. These are important as potential sites of current accumulation in the resistive fluid. In fact we can solve the ideal version of the induction equation $(\eta=0)$ along the azimuthal rays given by $\theta=k \pi / m+\phi$. In this region

$$
\sin (m \theta) \simeq(-1)^{k} m \phi, \quad \cos (m \theta) \simeq(-1)^{k},
$$

and if we assume $r \gg(2 \mu)^{1 /(2-m)}$, then the characteristic equations become

$$
\frac{d r}{(-1)^{k} \mu r^{m-1}}=-\frac{d \phi}{(-1)^{k} \mu m r^{m-2} \phi}=-\frac{d Z}{Z} .
$$

The characteristics are given by

$$
\xi=r \phi^{1 / m},
$$

and the ideal solution is

$Z=Z_{0}\left[r\left(\frac{\phi}{\phi_{c}}\right)^{1 / m}\right] \exp \left\{\frac{(-1)^{k}}{\mu(m-2)} r^{2-m}\left[1-\left(\frac{\phi_{c}}{\phi}\right)^{1-2 / m}\right]\right\}$,

where we have specified $Z\left(r, k \pi / m+\phi_{c}\right)=Z_{0}(r)$. The exponential blows up as $\phi \rightarrow 0$ for odd $k$ if $\mu>0$, and for even $k$ if $\mu<0$. In other words, the singularities only occur along the half planes that correspond to the fan surfaces of the outer nulls. To witness the field localization in practice, we must specify a positive null, i.e., $\alpha<0$, so that the central spine forms an outflow jet. There is, however, inflow along the spines of all satellite nulls.

\subsection{Resistive Solutions}

Because of the complexity of the induction equation (18), we must use numerical methods to explore the current build-up in the presence of resistive effects. The analytic problem has an open geometry, but we must assume a finite region (here we take the unit square) in constructing a numerical solution. Since the behavior of the solution on the boundary is unknown, we employ a numerical code that adopts boundary conditions based on the gross symmetries of the disturbance field. In all cases we find that an interior solution develops that is insensitive to the exact boundary values, except in a narrow transition layer close to the walls. The diagrams given below display only the interior solution. 

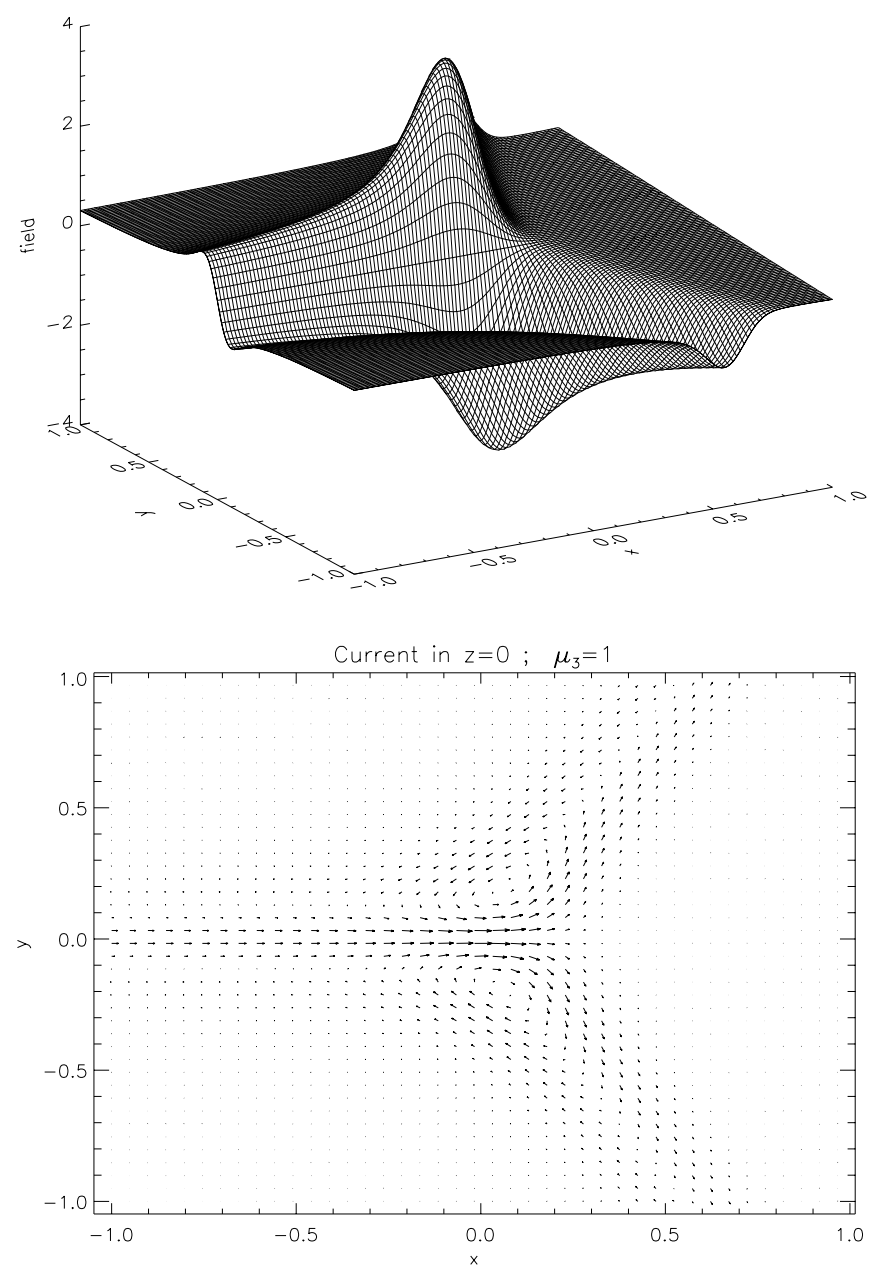

FIG. 3.-Field and current solutions for the background field (eq. [19]) with $m=3$ and $\eta=0.01$. Note how despite the presence of three nulls a distance $r=\frac{1}{2}$ along the arms, the majority of current still accumulates at the central spine.

Consider the $m=3$ solution discussed above. As shown in $\S 3.4$, we expect strong currents to develop on the fan surfaces of the of the outer nulls. In general we expect (see $\S 4.3$ ) intense current structures to be associated with a small $\eta^{1 / 2}$ length scale (see also $\S 3.2$ ). If we specify antisymmetry across the line $y=0$, a special role is assigned to the satellite null on the shear line $y=z=0$. As Figures $3 a$ and $3 b$ indicate, this null "attracts" more intense current structures than the two displaced nulls. Even so, the current density is strongest where the satellite fans meet, that is, along the spine axis of the central null.

These results are not surprising when we consider the separatrix structure of Figure 2. The disturbance field perturbs the fan of the central null, and so we expect spine currents to develop near the origin. The null at the point $x=1 /(2 \mu), y=0$ has its spine perpendicular to the shear axis, and so we anticipate strong fan currents in the region $x<0,|y| \leq \eta^{1 / 2}$. By the same token, the remaining satellites develop somewhat weaker fan currents since their spines are not perpendicular to the axis of shear.

Although, by systematically varying the resistivity, we can deduce scaling laws for the current density, we postpone a detailed discussion of resistive scalings until $\S 4$. We simply mention that the extended current structures brought about by the inclusion of a single ring of satellite

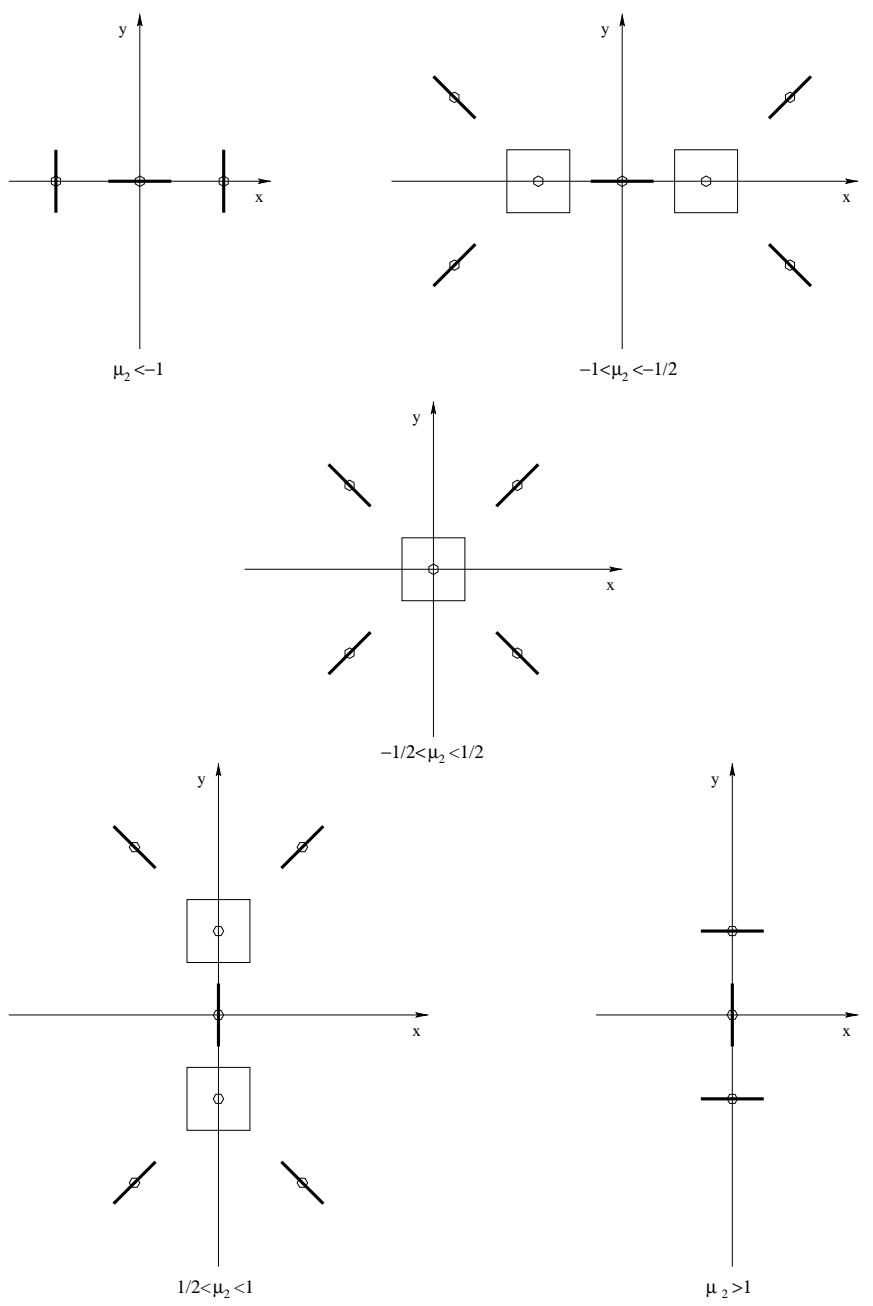

FIG. 4.-Schematic diagrams of the five different null topologies attainable by varying $\mu_{2}$. The small hexagons represent the locations of the nulls, while squares and thick lines represent fan surfaces parallel and perpendicular to the page, respectively.

nulls is not sufficient to compromise the energetically unfavorable spine-current scalings of the single null model. That is, although both spine and fan currents are present in the solution, it is the spine current at the origin that dominates in the limit of small resistivity. Apparently, more complexity in the field is required to achieve significantly new scalings.

\section{EXAMPLE OF A COMPLEX MULTINULL MODEL}

\subsection{Noncircular Symmetric Fields}

It appears that the addition of a single higher order term in the background field equation (19) does not lead to significant departures from the resistive scaling laws of the isolated null spine current model. Here we show that when the circular symmetry of the background field is relaxed, multiple null models can provide a range of solutions incorporating forms of fan, spine, and separator reconnection. An interesting feature of these models is the tendency of the current to be localized to separatrix structures of finite extent. Of course, currents sheets in single null models are always unbounded.

Suppose we eliminate the circular symmetry by combining the $m=2$ and $m=4$ background field components 
from equation (17). If we take

$$
\begin{aligned}
& P_{1}=r / 2+\mu_{2} r \cos (2 \theta)+\mu_{4} r^{3} \cos (4 \theta), \\
& P_{2}=-\mu_{2} r \sin (2 \theta)-\mu_{4} r^{3} \sin (4 \theta), \\
& P_{3}=-z,
\end{aligned}
$$

the Cartesian form of the field is

$$
\begin{aligned}
& P_{1}=\frac{x}{2}+x\left(\mu_{2}+\mu_{4} x^{2}-3 \mu_{4} y^{2}\right), \\
& P_{2}=\frac{y}{2}+y\left(-\mu_{2}-3 \mu_{4} x^{2}+\mu_{4} y^{2}\right), \\
& P_{3}=-z .
\end{aligned}
$$

We focus exclusively on the case $\mu_{4}>0$ when this field may contain as many as seven nulls. The parameter $\mu_{4}$ then determines an overall scale for the problem, while the parameter $\mu_{2}$ governs the number and structure of the nulls. The field morphology is illustrated in Figure 4.

We note that for $\left|\mu_{2}\right|>1$ there are only three colinear nulls, specifically, two outer nulls distributed symmetrically about the central null at the origin. When $\left|\mu_{2}\right|=1$ the two outer nulls each undergo a pitchfork bifurcation, generating an additional four satellite nulls to give a total of seven nulls. When $\left|\mu_{2}\right|=\frac{1}{2}$ the two innermost satellite nulls coalesce with the central null (again in a pitchfork bifurcation) to yield a five null configuration; see the Appendix for a detailed summary of the eigenstructure. Note the appearance of separator field lines joining the three inner nulls in the seven null case $\frac{1}{2}<\left|\mu_{2}\right|<1$. Changing the parameter $\mu_{4}>0$ has no effect on the structure of the nulls, but it does control the overall scale of the field. In what follows we choose $\mu_{4}$ so that the innermost satellite nulls always lie at $x= \pm \frac{1}{2}$. Figure 5 shows detailed field line plots for the three basic forms of background field in the plane $z=0$.

\subsection{The Disturbance Field}

We consider only the simple case of a disturbance field $Z(x, y)$ whose amplitude is independent of $x$ on the outer boundary. This corresponds to a global shearing of the background field across the $x$-axis. Since exactly the same results can be recovered by changing the parity of $\mu_{2}$ and shearing across the $y$-axis, it is clear that only the modulus of $\mu_{2}$ is essential.

Figure 6 shows the form of the disturbance field $Z$ and the resulting current density for the three, seven, and five null background fields discussed above. The resistive scaling of the current density at the central null is shown in Figure 7. Note that in Figure $7 b$ we see current spikes forming around the spines of the two outer nulls, which clearly scale at a faster rate than the current at the central
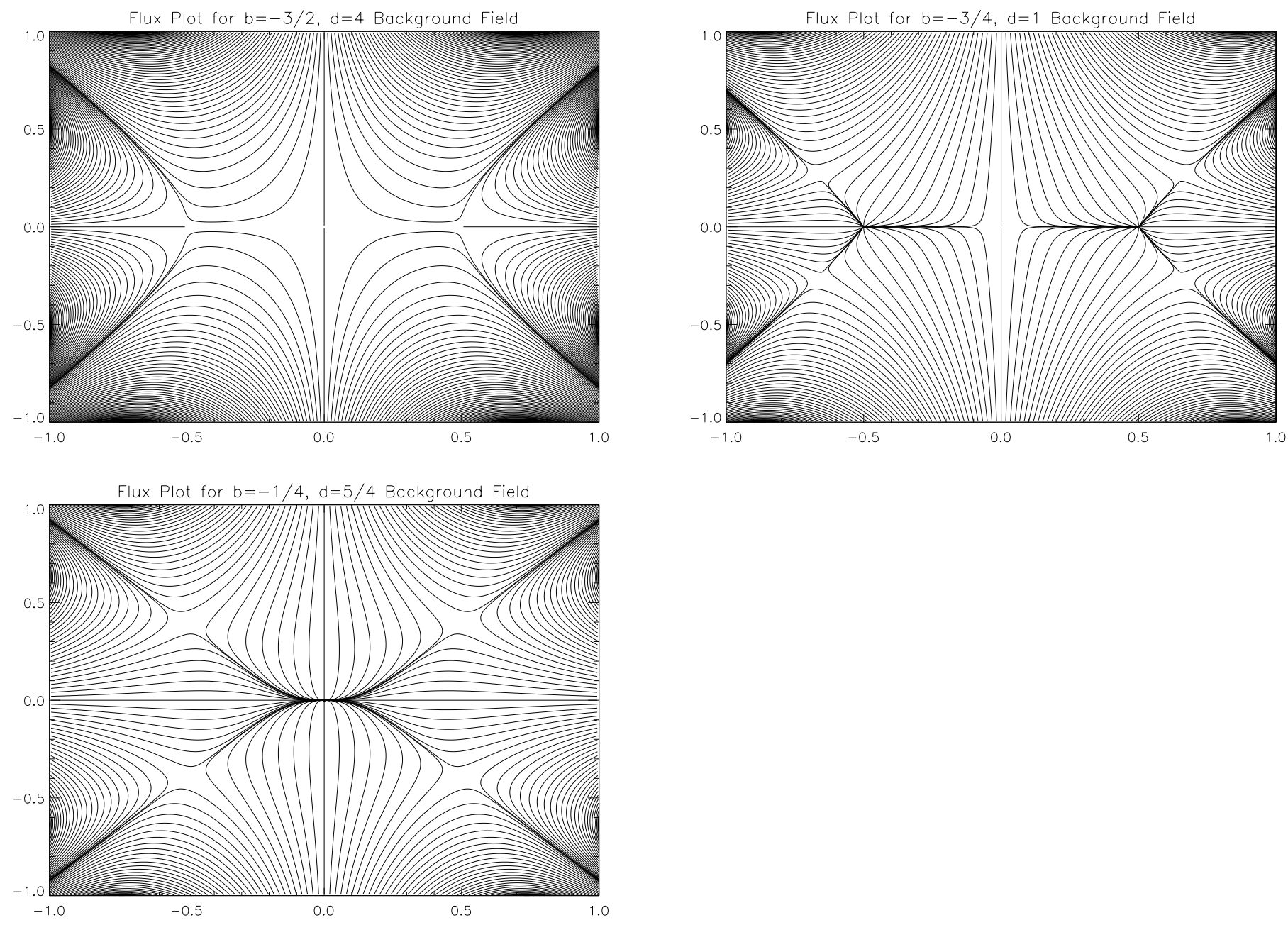

FIG. 5.-Diagrams of the field line structure in the $z=0$ plane for the first three different null configurations shown in Fig. 4 

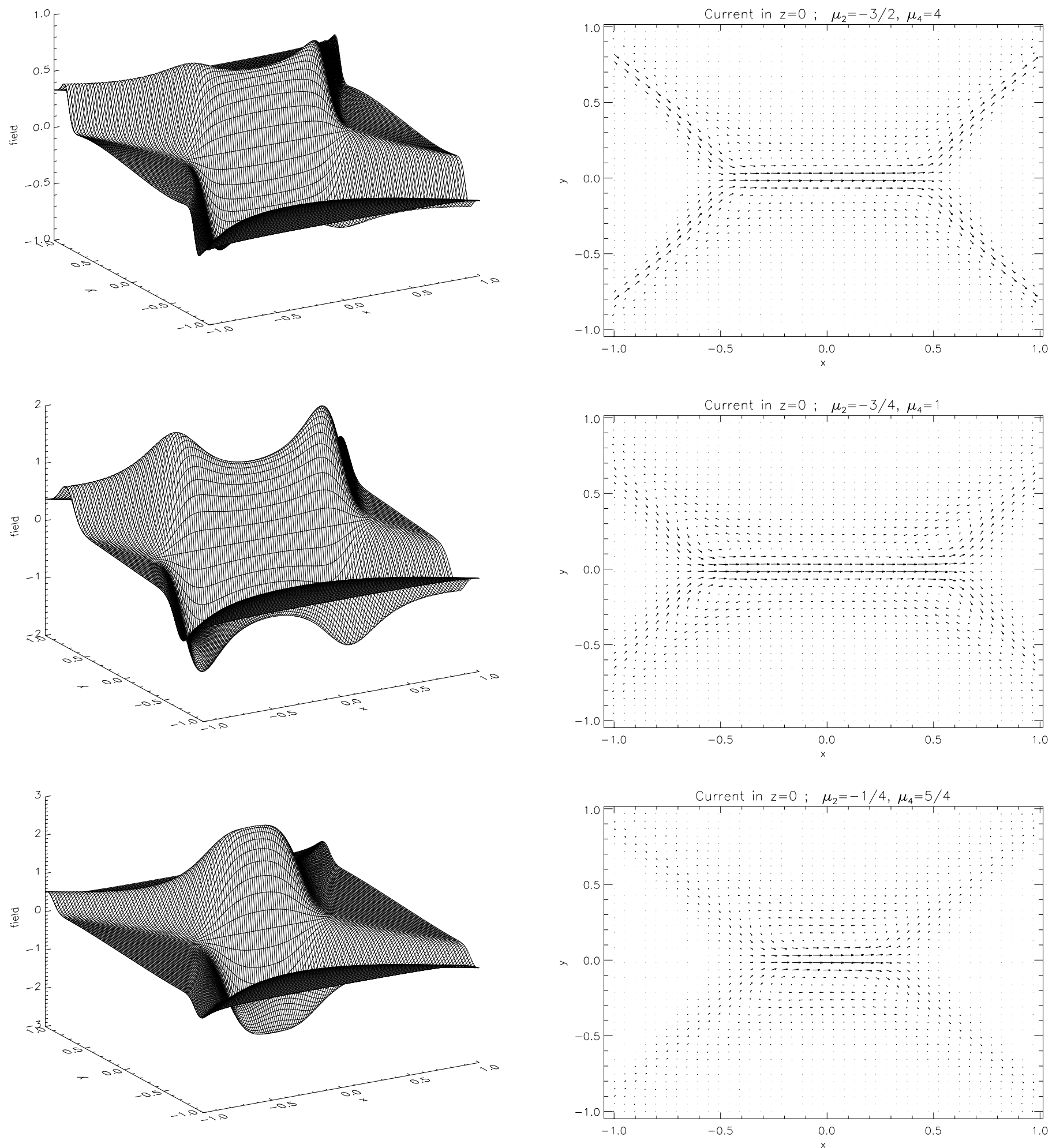

FIG. 6.-Numerical solutions of the induction eq. (11): field $Z(x, y)(l e f t)$ along with corresponding current (right). Note that the currents are only ever in planes of constant $z$ since these are perpendicular to the disturbance field.

null. Although the resistive scalings reflect the spine and fan structures of the background field, there is always a strong current localization along the $x$-axis close to the central null. It is not difficult, as shown below, to predict the detailed current scalings by assuming that the disturbance field has the form $Z=Z(y)$ in the vicinity of the central null. These analytic predictions are shown by the dashed lines in Figure 7.
Finally we emphasize that the disturbance field has the following interpretation (Watson \& Craig 1998): For the case $\lambda=0$ the disturbance field $Z(x, y) \hat{z}$ is the complete solution for the magnetic field of a nonreconnective annihilation model. If $\lambda$ is set nonzero, then $\boldsymbol{B}$ is formed by superposing $Z(x, y) \hat{z}$ with the background field $\boldsymbol{P}$ to give a fully reconnective solution. For example, to determine a separator reconnection model, we should superpose the back- 

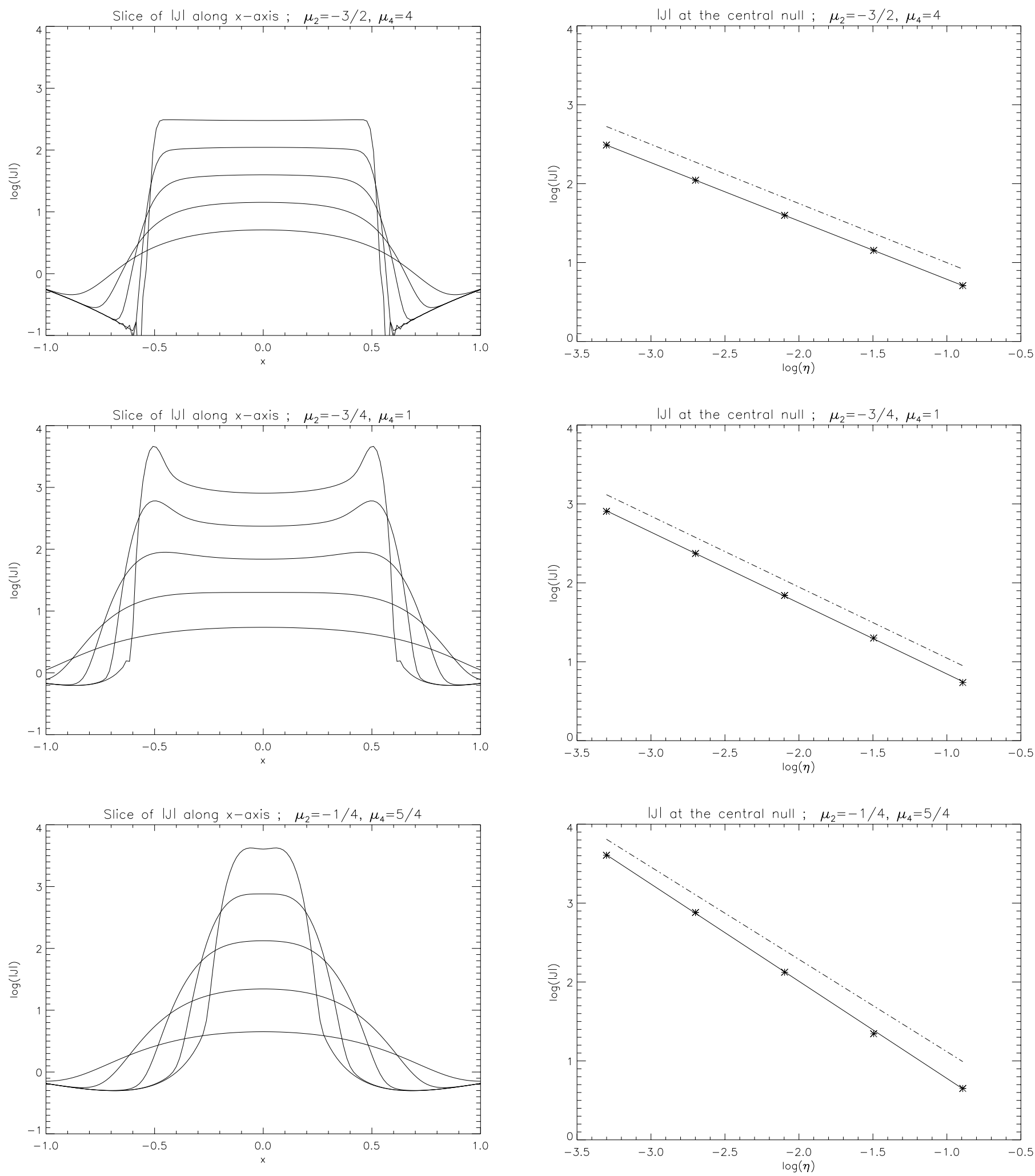

FIG. 7.- Montage of current magnitude along the $x$-axis (left), along with the scaling relation at the central null (right). The current plots are for $\eta=0.0005,0.002,0.008,0.032$, and 0.128 , with smaller values of $\eta$ generating larger currents. The dashed lines on the scaling plots represent the slope predicted by equation (23). Note the problem is slightly under resolved in the current plots for $\eta=5 \times 10^{-4}$.

ground field shown in Figure $5 b$ with the disturbance field given in Figure $6 b$.

\subsection{Analytic Scalings Using Boundary Layer Analysis}

Suppose we assume that $Z=Z(y)$ close to the central null. The leading order terms in the Cartesian form of the induction equation (11) reduce to

$$
\bar{\eta} Z^{\prime \prime}-\left(\frac{1}{2}-\mu_{2}\right) y Z^{\prime}-Z=0, \quad Z=Z(y),
$$

and we recover the presence of a small length scale $\Delta y \sim$ $|\bar{\eta}|^{1 / 2}$. If we identify the region $y<\Delta y$ as a resistive current layer, we can deduce resistive scaling laws based on a simple 
Central Null Current Maximum Scaling, exponent of $\eta$

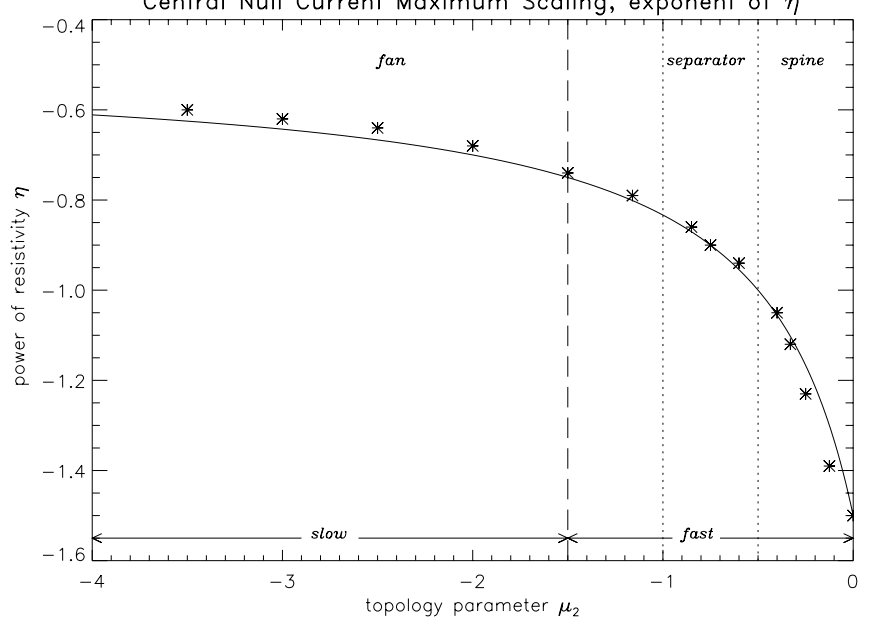

FIG. 8.-Summary of scalings for a range of $\mu_{2}$ values. The solid line is the scaling law as predicted by equation (23), while the asterisks mark values obtained by solving equation (11) numerically. The fan, separator, and spine regimes are separated by vertical dotted lines. Note that we have "fast" reconnection for $\mu_{2}>-3 / 2$.

boundary layer treatment of the induction equation. In the following analysis $\mu_{2}$ is taken to be negative. Identical results apply for positive $\mu_{2}$, provided we take $Z \rightarrow Z(x)$ and apply the shearing across the $y$-axis.

In the far field $y \gg \Delta y$ we have that

$$
\left(\mu_{2}-\frac{1}{2}\right) y Z^{\prime}=Z \text {, }
$$

and so

$$
Z=y^{2 /\left(2 \mu_{2}-1\right)},
$$

on taking the normalization $Z(1)=1$. An appropriate inner solution is simply $Z \sim y$, where the amplitude is chosen to match the outer solution at the edge of the resistive current layer $y \simeq \Delta y$.

We are interested in the amplitude of the field and the current in the region $y \leq \Delta y \sim \eta^{1 / 2}$. These scale as

$$
Z \sim \eta^{1 /\left(2 \mu_{2}-1\right)}, \quad J \sim \eta^{\left(3-2 \mu_{2}\right) /\left(4 \mu_{2}-2\right)} .
$$

The dashed lines in Figure 7 confirm that the predicted scalings of the current density accord very well with the numerical solutions.

\subsection{Summary of Resistive Scalings}

In Figure 8 we summarize the scaling results as function of the topology parameter $\mu_{2}$. Recall that the central null goes from having a fanlike current structure for $\left|\mu_{2}\right|>1$ through a separator phase, where a current ribbon is centered on the intersection of two fans, and finally through a spinelike current phase when $0<\left|\mu_{2}\right|<\frac{1}{2}$. It is a remarkable fact that the scalings appear uncompromised by bifurcations in the null structure of the background field.

Which values of $\mu_{2}$ correspond to fast reconnection? We can easily check that the dissipation rate

$$
W_{\eta} \simeq \eta J^{2} \Delta y \sim \eta^{\left(2\left|\mu_{2}\right|-3\right) /\left(4\left|\mu_{2}\right|+2\right)}
$$

becomes independent of $\eta$ when $\left|\mu_{2}\right|=3 / 2$, and so models with $\left|\mu_{2}\right|>3 / 2$ are associated only with slow ohmic dissipation. In fact, the slow Sweet-Parker scaling $W_{\eta} \sim \eta^{1 / 2}$, is obtained only in the fan current limit $\left|\mu_{2}\right| \rightarrow \infty$. More generally, as shown in Figure 8, the region of potentially fast energy dissipation includes some fan current models, in the range $1<\left|\mu_{2}\right|<3 / 2$, as well as the totality of separator and spine solutions.

Can we claim that multiple null reconnection is more favorable than single null reconnection? On the basis of the present results we cannot claim any significant improvement on the energetic scalings of the isolated fan reconnection model (see Craig et al. 1997). However, we believe the multiple null models are more realistic. The magnetic field solutions now display a higher degree of complexity, possibly more typical of the fields in the active corona. The current structures that develop in the new models are also better behaved: they are confined to localized ribbons and no longer extend uniformly out to infinity, as they do for an isolated null.

Finally, we mention that the fast resistive scalings of the present solutions are an artifact of flux pile-up in the disturbance field. Flux pile-up can be avoided (as in the SweetParker limit $\left|\mu_{2}\right| \rightarrow \infty$ ) but only at the cost of slow dissipation. However, it is now understood that fast dissipation models must saturate nonlinearly depending on the amplitude of the disturbance field and the level of the resistivity (Craig \& Watson 1998). Nonetheless it appears that flux pile-up solutions are capable of appreciable flarelike energy release at physically plausible levels of the plasma resistivity (Watson \& Craig 1997b; Craig et al. 1997).

\section{CONCLUSIONS}

We have demonstrated that a range of three-dimensional, multiple null, reconnection solutions can be constructed using the spine formulation of $\S 2.3$. The essential idea is that reconnection is associated with the superposition of finite amplitude disturbances on multiple null equilibria. An encouraging feature of the analysis is that it confirms - and provides a mathematical description for - many of the qualitative features of magnetic reconnection deduced using purely kinematic arguments (e.g., Lau \& Finn 1990; Priest $\&$ Titov 1996). There is also some evidence from X-ray observations that strong current ribbons localize on the intersections of fan surfaces and spine and separator lines (i.e., the quasi-separatrix layers of Demoulin et al. 1996). The implication is that current sheets, tubes, and ribbons may provide universal signatures for all forms of magnetic reconnection.

Perhaps more surprisingly, it is possible to display the basic ingredients of fan, spine, and separator reconnection using a simple multiple null model whose morphology is specified by a single structure parameter $\mu_{2}$. The form the reconnection takes depends both on the properties of the background field $\boldsymbol{P}$ - this comprises a maximum of seven nulls in the example given in $\S 4$ and the symmetries of the disturbance field $Q=Z(x, y) \hat{z}$. We have shown, however, that by systematically varying the bifurcation parameter $\mu_{2}$, we can construct a continuum of reconnection solutions, characterized by distinct, analytically predictable, resistive scaling laws. Solutions in the range $0 \leq\left|\mu_{2}\right| \leq 3 / 2$ correspond to fast dissipation, that is, $W_{\eta} \geq \eta^{0}$. Notably, the classical Sweet-Parker dissipation rate, namely, $W_{\eta} \sim \eta^{1 / 2}$, is recovered only in the limit $\left|\mu_{2}\right| \rightarrow \infty$. This rate is formally the slowest available for the present multiple null solutions.

It should be stressed that we expect our findings to be largely independent of the idealizations and assumptions we introduce for analytic tractability. We know, for example, that our formulation is robust to the inclusion of timedependent and viscous effects (Craig \& Watson 1998). 
Perhaps the most severe requirement is the presence of an ignorable coordinate in the disturbance field $Z(x, y) \hat{z}$. One way around this is to employ, from the outset, a numerical treatment of the disturbance field. Although a numerical solution will undoubtedly lead to more complex reconnection models, we believe that it is unlikely to compromise the role of spines, fans, and separators as regions of current accumulation in magnetic merging solutions.

\section{APPENDIX}

Nulls can be classified according to the geometry of the field lines threading the neutral point, in other words, in terms of separatrices isolating topologically distinct regions of magnetic flux. We expand the magnetic field in the neighborhood of a null point located at $x_{0}$ according to

$$
B_{i}\left(x_{0}+d x\right) \simeq B_{i, j}\left(x_{0}\right) d x_{j},
$$

where $B_{i, j}$ is an element of the Jacobian matrix $\partial \boldsymbol{B} / \partial \boldsymbol{x}$. To lowest order the differential field line equation $\gamma d x=\boldsymbol{B}$ gives $B_{i, j} d x_{j} \simeq \gamma d x_{i}$. Hence we have the eigenvalue equation

$$
\left(B_{i, j}-\gamma I_{i j}\right) d x_{j}=0
$$

where $I_{i j}$ is the identity matrix. The eigenvectors $d x_{j}$ determine field line directions in the vicinity of the null. The eigensystem is generally complex, but the nature of the field can be used to obtain properties of the eigenvalues. The trace of $B_{i, j}$ vanishes because of the divergence free condition $\nabla \cdot \boldsymbol{B}=0$, and thus the eigenvalues must satisfy

$$
\gamma_{1}+\gamma_{2}+\gamma_{3}=0 \text {. }
$$

In the case of a vanishing current, $B_{i, j}$ is a symmetric matrix, since $J_{i}=\epsilon_{i j k} B_{k, j}$, and therefore the eigenvalues $\gamma_{i}$ must also all be real. Note that eigenvalues of like sign are associated with eigenvectors spanning the fan plane.

\section{A1. EIGENSTRUCTURE OF THE SIMPLE MULTINULL CYLINDRICAL FIELD}

The background field given by equation (19) possesses a central null at $r=z=0$, as well as $m$ satellite nulls lying in a ring determined by

$$
\sin (m \theta)=0, \quad r^{m-2}=-\frac{1}{2 \mu \cos (m \theta)}, \quad z=0 .
$$

As $\sin (m \theta)=0$, then $\cos (m \theta)= \pm 1$. Therefore for $\mu>0$ we require $\cos (m \theta)=-1$ and $\theta=(2 j+1) \pi / m, j=0,1,2, \ldots$ in order for $r$ to be real. Alternatively, if $\mu<0$ we require $\cos (m \theta)=1$ and $\theta=2 j \pi / m, j=0,1,2, \ldots$ The eigenstructure of this field, for the case $\mu>0$, is summarized in Table 1 .

\section{A2. EIGENSTRUCTURE OF THE COMBINED MULTINULL BACKGROUND FIELD}

For the background field given in equation (22) with $\mu_{4}>0$, there are nine possible locations for the nulls, given by $z=0$ :

$$
\begin{aligned}
& x=0, \quad y=0, \\
& x=0, \quad y= \pm \sqrt{\frac{1+2 \mu_{2}}{2 \mu_{4}}}, \\
& x= \pm \sqrt{\frac{-1-2 \mu_{2}}{2 \mu_{4}}}, \quad y=0, \\
& x= \pm \sqrt{\frac{1-\mu_{2}}{4 \mu_{4}}}, \quad y= \pm \sqrt{\frac{1+\mu_{2}}{4 \mu_{4}}} .
\end{aligned}
$$

\begin{tabular}{|c|c|c|}
\hline $\begin{array}{l}\text { Location of Null } \\
\qquad(r, \theta, z)\end{array}$ & Eigenvalues $\gamma_{i} / \alpha$ & Eigenvectors \\
\hline $\begin{array}{l}(0,0,0) \ldots \ldots \ldots \ldots \ldots \ldots \ldots \ldots \\
{\left[(2 \mu)^{1 /(2-m)},(2 j+1) \pi / m, 0\right] \ldots \ldots}\end{array}$ & $\begin{array}{c}\frac{1}{2}, \frac{1}{2},-1 \\
1-m / 2, m / 2,-1\end{array}$ & $\begin{array}{l}\hat{\boldsymbol{r}}, \hat{\theta}, \hat{z} \\
\hat{\boldsymbol{r}}, \hat{\theta}, \hat{z}\end{array}$ \\
\hline
\end{tabular}

TABLE 1

The Eigenstructure of the Multiple Null Background Field With Cylindrical Symmetry IN THE Vicinity OF THe Nulls

NoTE.-The background field possesses $m$ satellite positive nulls evenly distributed about a central negative null located at the origin. Note that the eigenvalues have been normalized with respect to the field strength $\alpha$. 
TABLE 2

The Eigenstructure of a Combined Multinull BaCKground Field

\begin{tabular}{|c|c|c|}
\hline $\begin{array}{l}\text { Location of Null } \\
\qquad(x, y, z)\end{array}$ & Eigenvalues $\gamma_{i} / \alpha$ & Eigenvectors \\
\hline $\begin{array}{l}\{0,0,0) \ldots \ldots \ldots \ldots \ldots \\
\left\{0, \pm\left[\left(-1+2 \mu_{2}\right) / 2 \mu_{4}\right]^{1 / 2}, 0\right\} \\
\left\{ \pm\left[\left(-1-2 \mu_{2}\right) / 2 \mu_{4}\right]^{1 / 2}, 0,0\right\} \ldots \ldots \ldots \ldots \ldots \ldots \ldots \ldots \ldots \ldots \\
\left\{ \pm\left[\left(1-\mu_{2}\right) / 4 \mu_{4}\right]^{1 / 2}, \pm\left[\left(1+\mu_{2}\right) / 4 \mu_{4}\right]^{1 / 2}, 0\right\} \ldots \ldots \ldots\end{array}$ & $\begin{array}{c}\frac{1}{2}+\mu_{2}, \frac{1}{2}-\mu_{2},-1 \\
2-2 \mu_{2},-1+2 \mu_{2},-1 \\
-1-2 \mu_{2}, 2+2 \mu_{2},-1 \\
\lambda_{1}, \lambda_{2},-1\end{array}$ & $\begin{array}{c}\hat{x}, \hat{y}, \hat{z} \\
\hat{x}, \hat{y}, \hat{z} \\
\hat{x}, \hat{y}, \hat{z} \\
\boldsymbol{w}_{1}, \boldsymbol{w}_{2}, \hat{z}\end{array}$ \\
\hline
\end{tabular}

NotE.-The sign of the primary and secondary nulls depends only on the magnitude of $\mu_{2}$.

Note that a maximum of only seven nulls can exist for any fixed value of $\mu_{2}$ (see Fig. 4). The eigenstructure is summarized in Table 2. The fan planes of the nulls given in the last row of the table do not coincide with the coordinate axes. The eigenvalues and eigenvectors corresponding to these nulls are given by

$$
\lambda_{1}=\frac{1+\sqrt{1+8\left(1-\mu_{2}^{2}\right)}}{2}, \quad \lambda_{2}=\frac{1-\sqrt{1+8\left(1-\mu_{2}^{2}\right)}}{2}, \quad w_{1}=(1, A, 0), \quad w_{2}=(-A, 1,0),
$$

and

$$
A=\frac{\mu_{2}+\sqrt{1+8\left(1-\mu_{2}^{2}\right)}}{ \pm 3 \sqrt{1-\mu_{2}^{2}}}
$$

Craig, I. J. D., \& Fabling, R. B. 1996, ApJ, 462, 969

Craig, I. J. D., Fabling, R. B., \& Watson, P. G. 1997, ApJ, 485, 383

Craig, I. J. D., \& Henton, S. M. 1995, ApJ, 450, 280

Craig, I. J. D., \& Watson, P. G. 1998, ApJ, 516, 924

Demoulin, P., Mandrini, C. H., van Driel-Gesztely, L., Priest, E. R.,

Henoux, J. C., \& Schmieder, B. 1996, in ASP Conf. Ser. 111, Magnetic

Reconnection in the Solar Atmosphere, ed. R. Bentley \& J. Mariska (San

Francisco: ASP), 259

Fabling, R. B. 1997, Ph.D. thesis, Univ. Waikato

Fabling, R. B., \& Craig, I. J. D. 1996, Phys. Plasmas, 3, 2243

Forbes, T. G., \& Priest, E. R. 1987, Rev. Geophys., 25, 1587

Greene, J. M. 1988, J. Geophys. Res., 93, 8583

Lau, Y. T., \& Finn, J. M. 1990, ApJ, 350, 672

\section{REFERENCES}

Longcope, D. W. 1998, in Observational Plasma Astrophysics, ed. T. Watanabe (Dordrecht: Kluwer), 179

Longcope, D. W. \& Cowley, S. C. 1996, Phys. Plasmas, 3, 2885

Parker, E. N. 1979, Cosmical Magnetic Fields (Oxford: Clarendon)

Parnell, C. E. 1996, in ASP Conf. Ser. 111, Magnetic Reconnection in the Solar Atmosphere, ed. R. Bentley \& J. Mariska (San Francisco: ASP), 19 Priest, E. R. 1997, Phys. Plasmas, 4, 1945

Priest, E. R., \& Titov, V. S. 1996, Philos. Trans. R. Soc. London A, 354, 2951

Watson, P. G., \& Craig, I. J. D. 1997a, Phys. Plasmas, 4, 101 1997b, Phys. Plasmas, 4, 110 -.1998, ApJ, 505, 363 